\title{
Neonatal Resuscitation at the Peripheral Clinic
}

\author{
J.V. Larsen M.B. Ch.B., M.R.C.O.G., \\ Department of Obstetrics and Gynaecology, King Edward VIII Hospital.
}

\section{OPSOMMING}

Neonatale resussitasie in die kliniese situasie word in besonderhede bespreek. Die belangrikheid van behoorlike antenatale keuring van pasiënte vir kliniese bevalling word beklemtoon, ten einde die geboorte van babas van lae APGAR- tellings in die omgewing te voorkom.

\section{INTRODUCTION}

$\mathbf{T}$ HE low APGAR baby delivered in a clinic presents the midwife with a formidable problem. The problem arises from the fact that clinics generally are poorly equipped for major resuscitation of the newborn. The problem is further compounded by the fact that the baby with a low APGAR score travels very badly. He becomes hypothermic easily, and is likely to die if moved in a cold ambulance without oxygen or adequate heating. Then too, midwives are generally poorly trained for the task of resuscitating babies suffering from severe asphyxia neonatorum, because they received their training in hospitals with the basic assumption that a medical officer is always in the background ready to take over the management of such problems. Finally, acceptance by the community of clinic-based obstetric services is largely dependent upon the clinics" achieving a nil perinatal mortality rate. The delivery of a gasping newborn who dies in the midwife's hands can do great damage to the reputation of the service.

\section{THE IMPORTANCE OF ANTENATAL SCREENING}

The three important causes of fetal distress and therefore asphyxia neonatorum, are hypoxia, excessive head compression, and intra-uterine infection, see Figs. I and II. A high proportion of patients in whom these three factors operate, can be detected during the antenatal period, and thus selected for hospital delivery.

For example, placental insufficiency, and therefore fetal distress, is predictably more common in pregnancies complicated by hypertension, diabetes, postmaturity, antepartum hacmorrhage, and intra-uterine growth retardation. The elderly primipara and the patient of more than 40 years of age also show a higher incidence of placental insufficiency.

Excessive head compression occurs in patients in whom there is cephalopelvic disproportion because the pelvis is small or the baby is of excessive size.
Meticulous history-taking and thoughtful examination of the antenatal patient at each visit, as well as careful pelvic assessment, and assessment of fetal size from 36 weeks onwards, should enable the midwife to detect all such patients and select them for hospital delivery.

Fetal distress due to syphilitic damage to the placenta can largely be prevented by doing the Wassermann reaction in every antenatal patient and treating positive reactors with penicillin. Patients in whom other causes of recurrent intrauterine infection (e.g. toxoplasmosis) produce fetal compromise can usually be detected by careful history-taking. All patients who have two or more first trimester abortions, a previous unexplained stillbirth or neonatal death, a previous mid-trimester abortion, or recurrent premature labours, should be referred as early as possible in the antenatal period for investigation, antenatal care from the hospital and hospital delivery.

Experience has shown that careful selection of patients in this way greatly reduces the incidence of asphyxiated births in the clinic.

\section{THE IMPORTANCE OF INTRAPARTUM MONITOR- ING}

If the midwife is to avoid delivering asphyxiated babies in a clinic, it is essential that she should detect any signs of fetal compromise as early as possible in the labour, in order that the patient can be timeously transferred to the base hospital. The signs of fetal compromise which she can use, are fetal heart changes, meconium staining of the liquor amnii, and excessive moulding of the fetal skull together with other signs of cephalopelvic disproportion.

This really does mean that the fetal heart in labour must be monitored half hourly, before, during and after contractions. Such monitoring must be given a high priority by the midwife whose duties in a busy clinic may involve her in attending to 
patients with minor ailments as well as looking after the patients in the labour ward.

It also means that the liquor amnii should be looked at in early labour. Any patient who has passed from the passive phase of labour i.e. her cervix is 4 or more centimetres dilated, should have the membranes ruptured, in order to inspect the liquor. Membranes should not be ruptured before $4 \mathrm{cms}$ dilatation for fear of doing so in a patient who is not in fact in labour. They should of course also not be ruptured in a patient with a high floating head because of hydramnios, or with a malpresentation, as such patients should in any case be transferred immediately to hospital.

Finally it means that the midwife must carefully feel for occipito-parietal and parieto-parietal moulding of the fetal skull every time she performs a vaginal examination on the patient in labour. Such moulding should be graded according to the method described by Philpott, ${ }^{1}$ and correlated with the amount of head palpable above the brim and the rate of cervical dilatation, in order to detect evidence of cephalopelvic disproportion as early as possible. The use of a graphic display of progress in labour is very important in such a task. ${ }^{2}$

All patients with abnormal fetal heart patterns, meconium staining of the liquor, especially in early labour, and evidence of cephalo-pelvic disproportion, including a second stage of longer than 30 minutes, should be urgently transferred for delivery in hospital where the best facilities for the resuscitation and care of the newborn are available.

\section{CLINIC RESUSCITATION OF THE HEALTHY NEW- BORN}

Meticulous antenatal and intrapartum care will help ensure that only vigorous babies with good (7-10) Apgar scores are delivered in the clinic. Resuscitation of such newborns constitutes no serious problem, but there are important details which require emphasis:

\section{Temperature Maintenance}

Newborns arrive in the world with a wet skin from their uterine incubator in which their bodv temperature has been comtortably maintained by the mother. They are suddenly precipitated into a room temperature which dry, clothed adults find comfortable but which for them is extremely cold. It is important to their survival that labour wards should be as warm as is comfortably permissible, and that the baby be dried and his body covered as soon as possible. If he is handed to his mother, her body will help to keep him warm.

\section{Clear the airway gently}

The mucosal lining of a newborn's naso-pharynx is delicate and easily damaged. Rough, prolonged suctioning can therefore produce ulceration and predispose to infection and oedema of the nasal mucosa which can lead to respiratory difficulty in a newborn baby who does not know how to breathe through his mouth. In addition, such suctioning is a major vagal stimulus and can provoke reflex apnoea. Suctioning should therefore be gentle and aim only at removal of mucus from nose"and pharynx, not at the stimulation of respiration. This latter aim is better achieved by flicking the heels.

\section{The Umbilical Stump}

This is an open wound with a poor blood supply, and must be treated as such. Thus the midwife should never blow on the abdomen of the newborn in order to stimulate him. She should always wash her hands and use sterile instruments and sterile ties in attending to the umbilicus.

\section{RESUSCITATION OF THE BABY WITH A LOW APGAR SCORE IN THE CLINIC}

It is unfortunately true that even when the clinic midwife's selection of patients is excellent, she will still be faced by the birth of the occasional baby with a low Apgar score. The majority of these will be born to unbooked patients or patients who have not co-operated in their antenatal care.

The basic principles of resuscitation of such babies are as follows:

\section{Be Prepared}

Set out below is a list of the basic equipment essential to the task of resuscitation of the new-born:

An oxygen cylinder with reduction valve, flow meter, tubing and connections. A spare, full oxygen cylinder should always be on the premises.

An infant's laryngoscope that works.

A suction source.

Catheters for oxygen administration and suctioning.

Translucent plastic bags $27 \times 50 \mathrm{~cm}$ in size.

A sterile $10 \mathrm{ml}$ syringe with needle.

A sterile $2 \mathrm{ml}$ syringe with needle.

Ampoules of $50 \%$ dextrose water and $8,5 \%$ Soda Bic.

A bag and mask suitable for intermittent positive pressure ventilation of a newborn is desirable. (Good patterns are marketed by Penlon and Ambu). If the midwife has been trained in intubation of the newborn, the necessary 12 and 14 F.G. (no 3 and no. 2,5) neonatal endotracheal tubes with adaptors should be available for her use.

\section{Recognise the baby needing active resuscitation rapidly}

It is essential to realise that the baby born with a very low Apgar score may leave the midwife only a minute or two in which to save his life. He can be recognised by feeling the pulse rate at the umbilicus and noting his response to suctioning. A pulse rate of under 100 beats per minute means significant asphyxia is present, and demands urgent action. A pulse rate of under 60 beats per minute means that the baby requires immediate intermittent positive pressure ventilation with pure oxygen.

\section{Oxygen Administration}

Oxygen is life-saving, but only if it is in the right place, and this is in the baby's lungs. His airways should be thoroughly but gently suctioned. If a lot of meconium is in the pharynx, this should be removed by suctioning under vision using the laryngoscope, ideally before the baby takes his first breath, in order to prevent the meconium aspiration syndrome. 
As soon as his airway is clear, if the baby is breathing and he is given 2 litres of oxygen through a nasal catheter, his condition will rapidly improve. If he is not breathing, it is essential to blow oxygen into his lungs. This can best be done in a clinic which is not equipped with a bag and mask, by:

(a) Mouh-10-mouth ventatation with added oxygen. To do this, the midwife places the oxygen catheter in the corner of her mouth and turns up the llow rate to $t$ litres per minute. She then holds the baby's mouth open, at the same time lifting the lower jaw forward in order to maintain a clear airway, with her right hand. Her left hand is employed pinching the baby's nose closed and supporting his head. A mixture of air and oxygen can now be blown into the baby's open mouth if the midwife applies her mouth to his. When the baby's lungs are expanded she removes her mouth to take a breath herself while he exhales.

(b) The nasal orygen catheter technique. To do this, the midwife raises the oxygen flow through a nasal catheter to 4-6 litres per minute. If both nostrils are then squeezed closed, and the baby's mouth intermittently closed with the jaw held forward, the pressure of the oxygen will expand his lungs. As soon as the lungs have expanded, the jaw should be released to allow him to exhale. Once he has done so, the mouth is again closed. Beware of over-inflating the lungs. Intermittent administration of oxygen in this way can be continued almost indefinitely with the sole disadvantage that the stomach blows up the oxygen as well if it has to be continued for some time. This carries with it the danger of vomiting, and the suction machine must always be available to prevent inhalation of vomitus.

If a bag, endotracheal tube and laryngoscope are available, intubation and ventilation with this equipment is obviously best, care being taken to avoid intubating the right main bronchus. It is important with either technique to start early, and to continue until the baby has a normal pulse rate and is pink. The midwife can then stop and stimulate the baby by tlicking his feet. If he remains apnoeic, she should continue as long as 20 minutes.

\section{Maintain the Baby's Body Temperature}

The baby with a low Apgar score will cool rapidly during resuscitation if he is left lying naked and wet in a room temperature which is comfortable for an adult. As his body cools, his oxygen requirement rapidly rises, and thus if optimal oxygenation cannot be rapidly established, his death is hastened. He should therefore be adequately covered as early as possible during resuscitation. Most coverings however, have the disadvantage that the midwife can no longer inspect the baby's chest movements and colour. A transparent plastic bag slipped over the baby"s feet up to his shoulders has the advantage of insulating the baby from the surrounding air while he can still be adequately inspected. It prevents loss of heat by evaporation and irradiation and is very cheap. Provided care is taken never lo cover the baby s face with the bag, it provides a sale method of maintaining his body temperature during transportation especially if the baby is held against the mother"s body. If used routinely in babies of low birth weight, or with low Apgar scores born in the clinic, plastic hags provide a very cheap method of maintaing the at risk newborn"s body temperature during the critical few hours after birth.

\section{Correct Acidosis and Hypoglycaemia}

Hypoxia often leads to a vicious circle as illustrated in Figure III

Because of the development of acidosis and hypoglycaemia, some babies still won't breathe even after prolonged intermittent positive pressure ventilation with adequate maintenance of their body temperature. It is essential in such babies to correct these two biochemical abnormalities. Acidosis can be corrected by giving half to $1 \mathrm{cc}$ of 8,5 percent Sodium Bicarbonate solution per estimated kilogram body mass intravenously. Hypoglycaemia is best corrected by diluting 50 percent dextrose water with sterile saline to a 20 percent solution and injecting 1-2 $\mathrm{ml}$ per kilogram of estimated body mass. The intravenous injection is most easily given by inserting a needle into the umbilical vein in the cord about $4 \mathrm{~cm}$ from the umbilicus. The umbilical vein is usually identified because it is the thickest and darkest of the vessels in the cord. Injection should be slow, and an artery forcep must be applied proximal to the site of injection to prevent subsequent haemorrhage.

\section{Reverse the effects of Pethidine if necessary}

If the patient received Pethidine within 2-3 hours of delivery, depression of the respiratory centre by this drug should be considered. It can always be rapidly corrected by giving Narcan Neonatal $2 \mathrm{mg}$ intramuscularly. There is no place for other respiratory stimulants ${ }^{3}$ such as Vandid and Nikethamide as these agents are now regarded as potentially dangerous

\section{Timing the transfer of the baby with a low Apgar score}

When resuscitation has been successful, it is important that the baby should be observed in an intensive care area in the base hospital for a few days. If he is progressively improving on oxygen in a warm place in the clinic, no attempt should be made to move him until optimal arrangements have been made to maintain his body temperature and oxygenation in the ambulance. This often means that it is better to move him the following morning when the sun is up than in a cold, poorly insulated ambulance at night when there is no portable incubator available. If he remains cynaosed and in a poor condition, urgent transfer on oxygen may however be the correct decision.

\section{Knowing when to stop}

If the baby fails to respond to all the resuscitative manoevres detailed above, intermittent positive pressure ventilation should be continued for 20 minutes. Should he not initiate sustained, spontaneous respiration by that time, it is almost certain that he has suffered irreversible cerebral damage and attempts to resuscitate him should probably be discontinued.

\section{LEARN FROM MISTAKES}

It is important to improving the standard of care in a clinic that every delivery complicated by the birth of a baby with a 
low Apgar score be discussed in detail with the supervising medical officer. The aim of such a discussion must be that of identifying avoidable factors and thus providing continuing education of the midwife in order that the objective of a nil clinic perinatal mortality can be obtained and maintained.
REFERENCES:

1. Philpott R.H. (1977) in Philpott R.H., Sapire K.E. and Axton J.H.M. Ed. "Obstetrics, Family Planning and Paediatrics", p 59. Natal University Press.

2. Ibid, p 57

3. Axton J.H.M. (1977) lbid, pg 256.

4. Neerhout R.C. and Sturgeon P. (1972) in Assali N.S. and Brinkman C.R. Ed "Pathophysiology of Gestation," pg 429, Academic Press: New York and London.

\section{FIGURE I}

\section{THE EFFECT OF INTRA-UTERINE INFECTION ON THE FETUS}

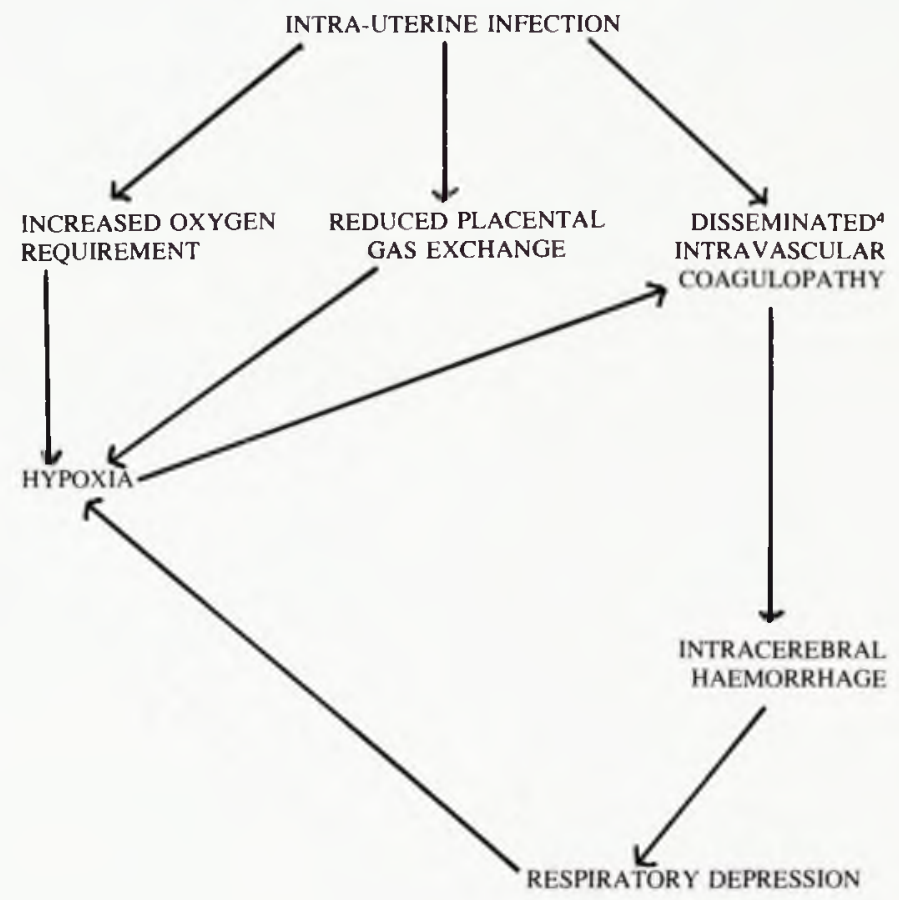

FIGURE II

THE EFFECT ON CEREBRAL TRAUMA ON THE NEONATE

FIGURE III

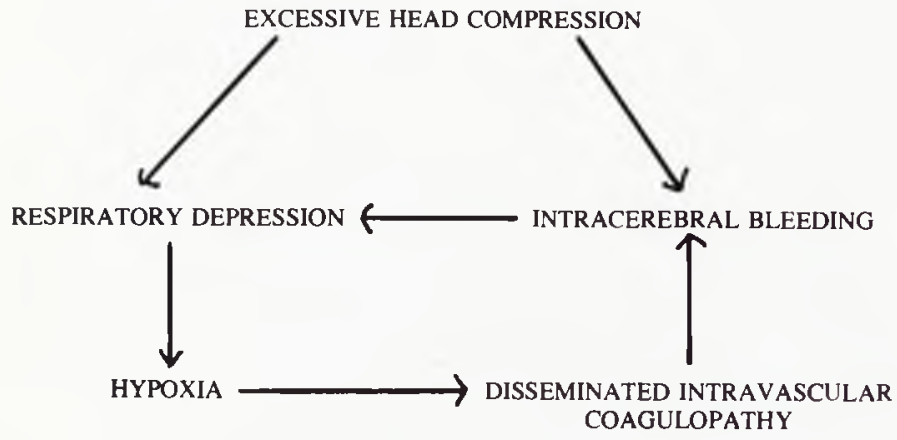

A SIMPLIFIED DIAGRAM OF THE EFFECTS OF HYPOXIA

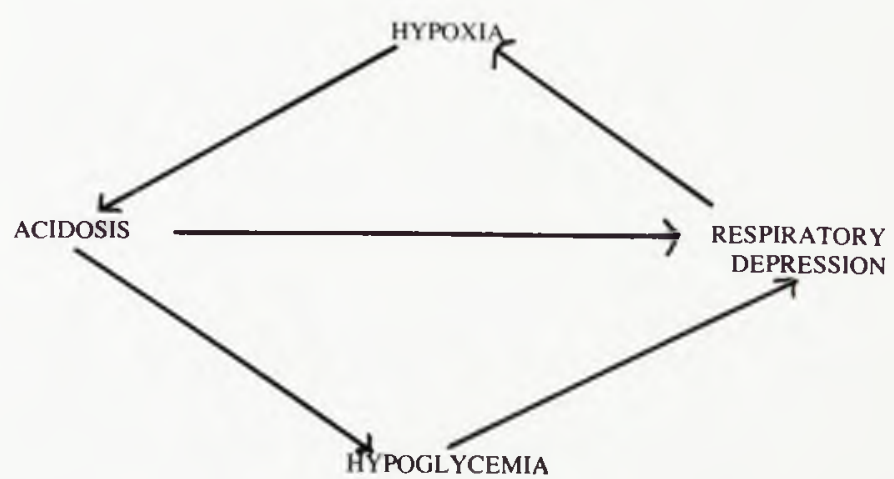

